\title{
44
}

\section{Adaptive QoS in Multimedia Systems}

\author{
M. Ott, G. Michelitsch, D. Reininger, G. Welling \\ C\&C Research Laboratories, NEC USA, Inc. \\ <http://www.ccrl.nj.nec.com> \\ max|georgldjr|welling@ccrl.nj.nec.com
}

\begin{abstract}
We propose the introduction of an abstract concept of QoS to all layers of the multimedia software architecture. Each layer in a software system deals with QoS at its appropriate level of abstraction using a generic API for communicating QoS parameters and values to layers above and below. We call the aggregation of these parameters and values a "service contract". By mapping the contract at one layer to other contracts at the layer below we can build service hierarchies that allow the design of adaptive systems based on a single framework. Further the API allows for reporting of contract violations as well as dynamic renegotiations of the contract terms.

To verify these ideas we built a proof-of-concept multimedia system (Ott, 1997) which showed that our architecture is not only feasible but offers a powerful framework for building adaptive multimedia systems.
\end{abstract}

\section{Keywords}

Multimedia, Distributed Systems, Quality of Service (QoS).

\section{INTRODUCTION}

The scalability of software systems within the bounds of available resources entails the notion of quality of service (QoS). Current systems address this notion either by hiding variations in available resources low down in the software hierarchy, or by implementing a resource allocation model based on hard call admission. However, we believe the usage patterns of current systems require QoS support at all levels of the software hierarchy. Further, a soft resource allocation model is essential for software systems aimed at the computing and communication environments of the future.

\section{ARCHITECTURE}

Most QoS architectures (Campbell, 1996) provide a QoS aware API to applications. This is either achieved by adding QoS parameters to standard system calls, or raising the system abstractions to a higher level, filling the gap with what is often referred to as middleware. In either case there seems to be a curious desire to draw a very strict 
line between "us", the QoS providers, and "them", the elusive applications. However, the same principles of modularity and abstractions found in network and system design are routinely used in applications as well. It therefore, seems natural to define an architecture which allows the introduction of QoS at any level: from CPU and network resources, to the user's "satisfaction".

In addition, the concept of "competing" applications often runs contrary to the way they are used. For instance, all applications on a terminal serve the same user, who may frequently change the relative importance of applications. In a resource limited environment such usage patterns can add in allocating a resource where it is needed most. Resources can be shifted from a less important application to one which currently has the user's focus. To support such behavior a mechanism is needed to shift resources between applications. It is obviously advantageous if the applications will actively cooperate in this process.

In order to realize the objectives described, we define a simple model as shown in Figure 1. A consumer and provider interact using a generic API which can be recursively applied to all levels of the software hierarchy. The consumer desires a service which is specified by a service contract. A provider for the service is located by some means, such as a third-party broker, and a binding is established. After the binding has been made, either party to the contract can initiate a renegotiation of the contract terms. Changes in QoS can therefore be initiated by the user according to his preferences (top down), or by the most primitive resource providers in order to change resource distribution. In case of a failed renegotiation, the consumer can also bind to a new service provider. For instance, a "local traffic" service may switch "city traffic" providers depending on the location of the user.

\subsection{Service Contract}

Part of the generic API is the abstract data type of a ServiceContract. It holds a set of QosParameters. It is the specifics of these parameters which completely describe the service. A parameter is a tuple of name, type, and value. These terms can either be used as requirements that a software component imposes

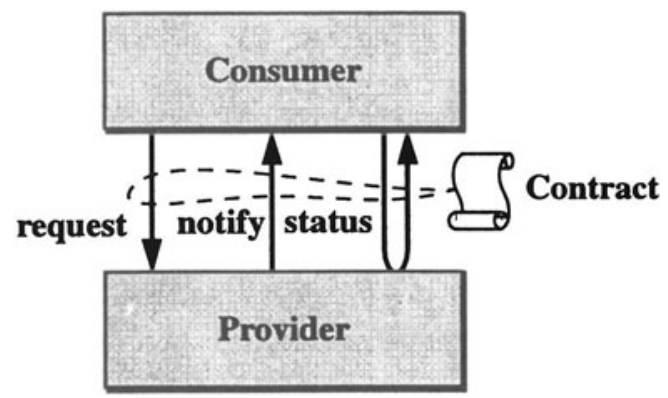

Figure 1 Consumer- provider model 
on a service, or as a measure of compliance of a service with its requirements.

A service provider can itself enlist the service of other software components creating a hierarchy of services. There can be a distinct service contract at each level using the same generic interface for contract negotiation. As shown in Figure 2, it will be very common for a consumer to request the service of multiple providers. In fact, most services will simply provide a "smart" mapping from their provider contract to multiple "consumer" sub-contracts.

Applied to multimedia systems; a video service providing movie $\mathrm{X}$ to user $\mathrm{Y}$ may sub-contract with a video server, a transport service, and a display station. These in turn will sub-contract to lower levels and will ultimately terminate in contracts for physical resources, such as CPU cycles, buffer space, and network capacity.

Also shown in Figure 2 is our notion of control and management. It may be possible to decompose a given contract into several different sets of sub-contracts. The main function of management is to evaluate these options and choose the most appropriate one. The control then attempts to satisfy the contract by fine-tuning the set of sub-contracts chosen. In case the control fails to do so management is notified. The management can now attempt to maintain contract compliance by trying a different set of sub-contracts. If all this fails the consumer of this service is notified and presented with a maintainable contract.

\subsection{Generic Interface for Contract Negotiation}

The generic API between any consumer and provider is shown Figure 1. It consists of three primitives necessary for service contract negotiations:

- Request: The consumer presents a contract to the provider.

- Notify: The provider presents the consumer with a new contract if the current one cannot be maintained.

- Status: Optionally, the consumer can query the provider about contract compliance.

The functionality of "request" and "notify" have been described above. However,

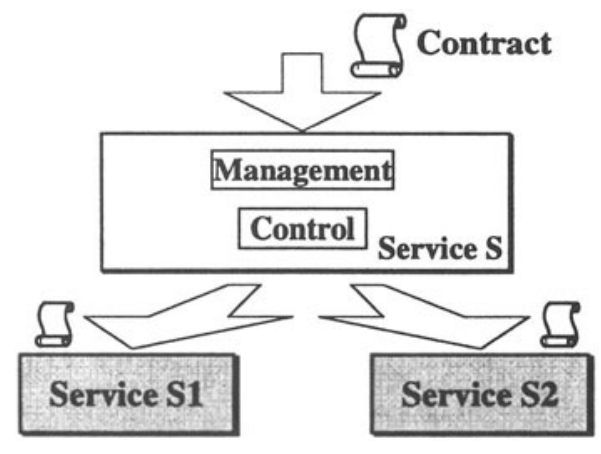

Figure 2 Service Hierarchy 
so far we have presented a passive consumer which only reacts to "notify" events. The "status" primitive allows a consumer to actively monitor contract compliance and possibly avoid "notify" calls by pre-emptive "requests" for altered contracts.

\section{EXPERIENCES}

We applied this approach to three important components of a typical distributed multimedia system (Ott 97). At the user interface level we show how limited screen real estate and the shifting focus during a typical machine user interaction offers ample opportunities for media tasks to change their resource demands. Within the network domain we show that "soft" bandwidth allocation based on media-specific "satisfaction profiles" is an important mechanism to balance the conflicting requirements of QoS and network utilization. The results show that a significantly better network utilization can be achieved when the adaptivity of the video source is integrated within the bandwidth allocation scheme. Finally, we applied the same architectural framework to the processor as a compute resource. The implementation of the proposed scheduler demonstrates that it is possible to decode and display several compressed video streams concurrently on an UltraSPARC-1 using software decoding. Under heavy load each video playback continues to run utilizing a proportional share of the CPU.

\section{CONCLUSION}

Based on our strong belief that future multimedia systems will be both distributed and heterogeneous, and the experience gained from building several multimedia prototypes in the past, we advocate an architectural framework for introducing adaptive QoS into every layer of such systems. Quality of service is specified by contracts which are established between clients and service providers using a single, generic API. The recursive application of this mechanism establishes a service hierarchy which constitutes QoS for the system as a whole.

During the course of experimenting with our prototype system, we observed the ease with which we could quickly build an application that could run under different environmental conditions. Clearly, our proposed architecture is generic and applicable to a variety of different resource domains. However, we are yet to fully test its power in building higher level abstractions.

\section{REFERENCES}

Campbell, A. et al. (1996) A Review of QoS Architectures, ACM Multimedia Systems Journal.

Ott, M. et al. (1997) An Architecture for Adaptive QoS and its Application to Multimedia Systems Design, <http://www.ccrl.nj.nec.com/papers/97-R-005/>. 